\title{
Hertz-level-linewidth semiconductor laser via injection locking to an ultra-high $Q$ silicon nitride microresonator
}

\author{
Warren Jin ${ }^{1, \dagger}$, Qi-Fan Yang ${ }^{2, \dagger}$, Lin Chang ${ }^{1, \dagger}$, Boqiang Shen ${ }^{2, \dagger}$, Heming Wang ${ }^{2, \dagger}$, Mark A. Leal ${ }^{1}$, \\ Lue $\mathrm{Wu}^{2}$, Maodong $\mathrm{Gao}^{2}$, Avi Feshali ${ }^{3}$, Mario Paniccia ${ }^{3}$, Kerry J. Vahala ${ }^{2, *}$, and John E. Bowers ${ }^{1, *}$ \\ These authors contributed equally to this work \\ ${ }^{1}$ ECE Department, University of California Santa Barbara, Santa Barbara, CA 93106, USA \\ ${ }^{2}$ T. J. Watson Laboratory of Applied Physics, California Institute of Technology, Pasadena, CA 91125, USA \\ ${ }^{3}$ Anello Photonics, Santa Clara, CA \\ vvahala@caltech.edu,bowers@ece.ucsb.edu
}

\begin{abstract}
A conventional semiconductor DFB laser is self-injection-locked to a CMOS-foundryfabricated ultra-high $Q$ silicon nitride microresonator, suppressing high-offset frequency noise to $0.2 \mathrm{~Hz}^{2} \mathrm{~Hz}^{-1}$ and yielding instantaneous linewidth of $1.2 \mathrm{~Hz}$. ๑ 2021 The Author(s)

OCIS codes: (130.3120) Integrated optics devices; (140.3945) Microcavities;
\end{abstract}

The integration of semiconductor lasers with high spectral purity and stability will be critical for future scalable coherent optical systems, including light detection and ranging (LIDAR) [1,2], laser gyroscopes [3,4], spectroscopy [5], optical frequency synthesis [6], optical atomic clocks [7], coherent communication networks [8], and microwave photonics [9-12]. A key ingredient for many of these systems is a mass-produced, low-noise laser. However, typical communication laser linewidths range from $100 \mathrm{kHz}$ to a few $\mathrm{MHz}$ [8], which is orders of magnitude too large for these applications. Applying optical feedback through an external reflector is a powerful method of quieting a laser [13-16]. Since the degree of noise suppression scales inversely with the optical linewidth of the reflector [15], ultrahigh- $Q$ microresonators are excellent candidates to achieve substantial linewidth narrowing and have been demonstrated across a wide range of materials as standalone [17] or integrated [18-20] components. Here, we harness CMOS-foundry fabrication to achieve an ultra-high intrinsic $Q$ of over $200 \mathrm{M}$ in a silicon nitride integrated photonic platform. We self-injection-lock an ultra-high $Q$ resonator chip to a conventional semiconductor distributed-feedback (DFB) laser, thereby reducing laser noise to a frequency noise of $0.2 \mathrm{~Hz}^{2} \mathrm{~Hz}^{-1}$ with corresponding instantaneous linewidth of $1.2 \mathrm{~Hz}--$ a previously unattainable level for integrated lasers.

$$
\text { a }
$$

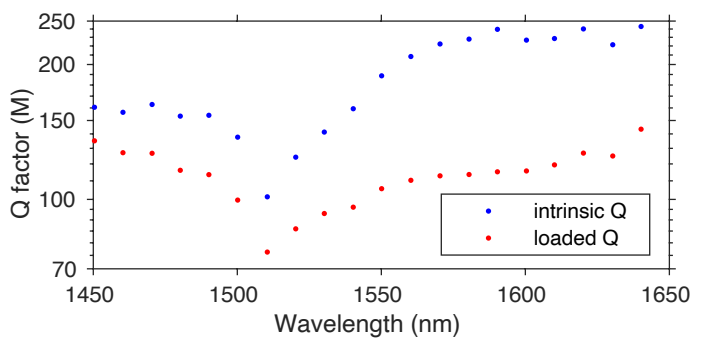

b

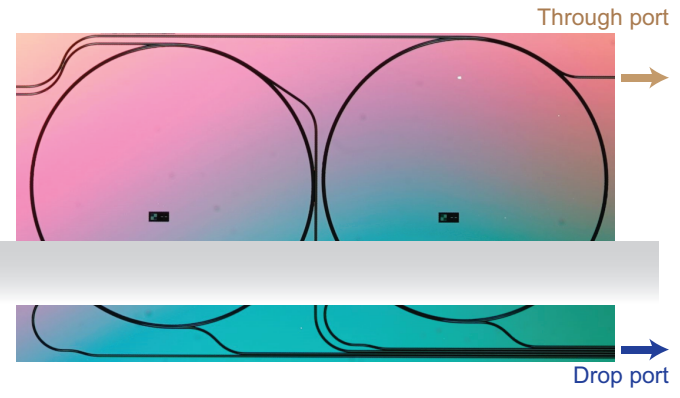

C

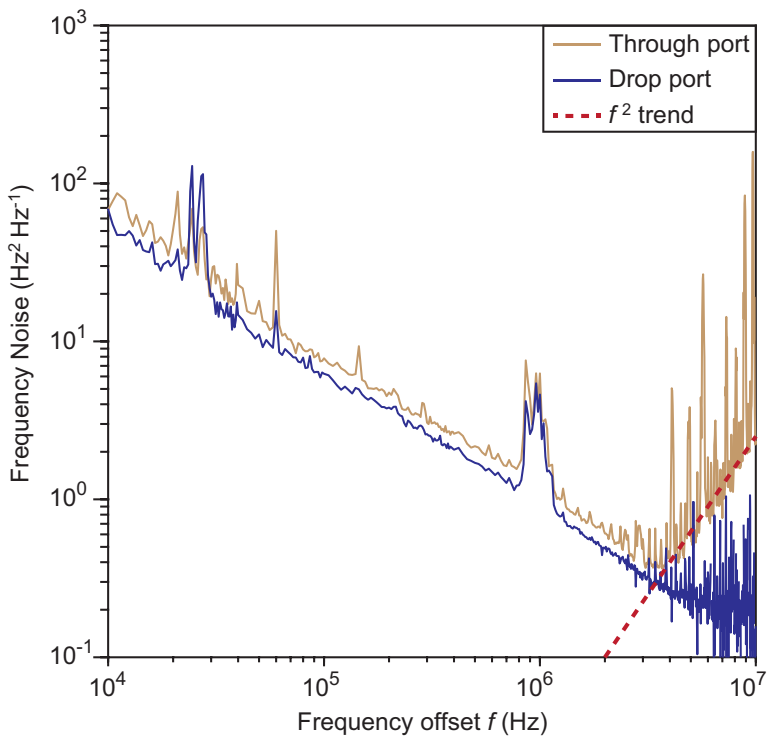

Fig. 1: (a) Intrinsic and loaded $Q$ factors of a ring resonator featuring a drop port, measured at various wavelengths. (b) Optical micrograph of the fabricated ring resonator with $10.8 \mathrm{GHz} F S R$. (c) Frequency noise of the DFB laser operating at $1556 \mathrm{~nm}$ when self-injection-locked to the resonator, measured from the through port and drop port. 
The waveguide geometry of the ultra-high $Q$ resonator consists of a $100 \mathrm{~nm}$ thick silicon nitride core embedded within $14.5 \mu \mathrm{m}$ silicon dioxide lower cladding, and $2.2 \mu \mathrm{m}$ of silicon dioxide upper cladding. A waveguide width of $2.8 \mu \mathrm{m}$ allows single mode propagation, however within the resonator itself the waveguide width is $25 \mu \mathrm{m}$ to minimize sidewall scattering losses. The coupler is designed to access the fundamental TE mode of the resonator, which takes the form of a whispering-gallery mode. Additionally, the resonator is fabricated with two sets of couplers, allowing the optical output to be taken from either the through port or the drop port. The device is fabricated on a $200 \mathrm{~mm}$ diameter silicon wafer within a high-volume CMOS foundry. The intrinsic and loaded Q factors of the device are shown in Fig. 1(a), demonstrating that an intrinsic $Q$ factor over $200 \mathrm{M}$ and loaded $Q$ factor over $100 \mathrm{M}$ is achieved for wavelengths longer than $1550 \mathrm{~nm}$. An optical micrograph of the fabricated device is shown in Fig. 1(b). A commercial DFB laser is butt-coupled to the bus waveguide of the silicon nitride chip. The laser is able to deliver power up to $120 \mathrm{~mW}$ at $1556 \mathrm{~nm}$. The optical feedback is provided by backward Rayleigh scattering in the microresonator, which reaches its maximum once the laser is on-resonance. Such feedback spontaneously aligns the laser frequency to the closest mode, where the phase accumulated in the feedback is critical to determining the stability of injection-locking. In our experiment, the feedback phase is precisely controlled by adjusting the air gap between the laser chip and the bus waveguide. In the case of a rigidly co-packaged laser and resonator, feedback phase control could instead be achieved by the addition of a resistive heater to the waveguide linking the laser and resonator. The laser output is taken from either the through port or drop port of the resonator as indicated within Fig. 1(b).

The measured frequency noise of the self-injection-locked laser system is shown in Fig. 1(c). For laser output taken at the through port, the frequency noise is observed to reach a minimum at $4 \mathrm{MHz}$ offset frequency, beyond which the frequency noise begins to rise at a rate proportional to the square of offset frequency. This is because the maximum noise suppression bandwidth in an injection locking system is limited to the bandwidth of the resonator [15]. To achieve an ultra-low white frequency noise floor at high offset frequency, the laser output is instead taken from the drop port of the resonator. The drop port provides low-pass filtering action and yields a white-noise floor of $0.2 \mathrm{~Hz}^{2} \mathrm{~Hz}^{-1}$ with corresponding instantaneous linewidth of $1.2 \mathrm{~Hz}$.

This work is supported by the Defense Advanced Research Projects Agency, DARPA (HR0011-15-C-055, FA9453-19-C-0029) and Anello Photonics.

[1] Trocha, P. et al. Ultrafast optical ranging using microresonator soliton frequency combs. Science 359, 887-891 (2018).

[2] Suh, M.-G. \& Vahala, K. J. Soliton microcomb range measurement. Science 359, 884-887 (2018).

[3] Gundavarapu, S. et al. Sub-hertz fundamental linewidth photonic integrated Brillouin laser. Nat. Photon. 13, 60- 67 (2019).

[4] Lai, Y.-H. et al. Earth rotation measured by a chip-scale ring laser gyroscope. Nat. Photon. 14, 345-349 (2020).

[5] Suh, M.-G., Yang, Q.-F., Yang, K. Y., Yi, X. \& Vahala, K. J. Microresonator soliton dual-comb spectroscopy. Science 354, 600-603 (2016).

[6] Spencer, D. T. et al. An optical-frequency synthesizer using integrated photonics. Nature 557, 81-85 (2018).

[7] Newman, Z. L. et al. Architecture for the photonic integration of an optical atomic clock. Optica 6, 680-685 (2019).

[8] Kikuchi, K. Fundamentals of coherent optical fiber communications. J. Light. Technol. 34, 157-179 (2015)

[9] Li, J., Lee, H. \& Vahala, K. J. Microwave synthesizer using an on-chip brillouin oscillator. Nat. Commun. 4, 2041-1723 (2013).

[10] Liang, W. et al. High spectral purity Kerr frequency comb radio frequency photonic oscillator. Nat. Commun. 6, 7957 (2015).

[11] Marpaung, D., Yao, J. \& Capmany, J. Integrated microwave photonics. Nat. Photon. 13, 80-90 (2019).

[12] Liu, J. et al. Photonic microwave generation in the Xand K-band using integrated soliton microcombs. Nat. Photon. 14 (2020).

[13] Liang, W. et al. Ultralow noise miniature external cavity semiconductor laser. Nat. Commun. 6, 1-6 (2015).

[14] Kondratiev, N. et al. Self-injection locking of a laser diode to a high-Q WGM microresonator. Opt. Express 25, 28167-28178 (2017).

[15] Li, H. \& Abraham, N. Analysis of the noise spectra of a laser diode with optical feedback from a high-finesse resonator. IEEE J. Quantum

Electron. 25, 1782-1793 8 (1989).

[16] Shen, B. et al. Integrated turnkey soliton microcombs. Nature 582, 365-369 (2020).

[17] Lee, H. et al. Chemically etched ultrahigh-Q wedge resonator on a silicon chip. Nat. Photon. 6, 369-373 (2012).

[18] Spencer, D. T., Bauters, J. F., Heck, M. J. \& Bowers, J. E. Integrated waveguide coupled Si3N4 resonators in the ultrahigh-Q regime. Optica 1, 153-157 (2014).

[19] Ji, X. et al. Ultra-low-loss on-chip resonators with submilliwatt parametric oscillation threshold. Optica 4, 619- 624 (2017).

[20] Liu, J. et al. High-yield wafer-scale fabrication of ultralow-loss, dispersion-engineered silicon nitride photonic circuits. arXiv preprint arXiv:2005.13949 (2020). 